AperTO - Archivio Istituzionale Open Access dell'Università di Torino

\title{
Objective Coordination with Business Artifacts and Social Engagements
}

\section{This is the author's manuscript}

Original Citation:

Availability:

This version is available http://hdl.handle.net/2318/1645303

since 2018-07-11T22:16:45Z

Publisher:

Springer

Published version:

DOI:10.1007/978-3-319-74030-0

Terms of use:

Open Access

Anyone can freely access the full text of works made available as "Open Access". Works made available under a Creative Commons license can be used according to the terms and conditions of said license. Use of all other works requires consent of the right holder (author or publisher) if not exempted from copyright protection by the applicable law. 


\title{
Objective Coordination with Business Artifacts and Social Engagements
}

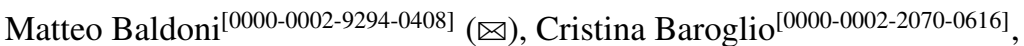 \\ Federico Capuzzimati ${ }^{[0000-0003-4023-6379]}$, Roberto Micalizio ${ }^{[0000-0001-9336-0651]}$ \\ Università degli Studi di Torino — Dipartimento di Informatica \\ c.so Svizzera 185, I-10149 Torino (Italy) \\ \{firstname.lastname\}@unito.it
}

\begin{abstract}
This work studies business artifacts by tackling a limit that we see in the current model, which is: business artifacts are not devised as natural means of coordination in their own right, despite the fact that they have the potential of being natural means of coordination in their own right. Coordination issues are transfered (e.g. by BALSA) to solutions that are already available in the literature on choreography and choreography languages. Instead, we propose to enrich business artifacts with a normative layer that accounts for the social engagements of the parties which interact by using a same business artifact. We explain the advantages, also from a software engineering perspective, and propose an approach that relies on the notion of social commitment.
\end{abstract}

Keywords: Business Artifacts, Normative MAS, Social Commitments

\section{Introduction}

The artifact-centric approach $[6,12,10]$ is recently emerging as a viable solution for specifying and deploying business operations by combining both data and processes as first-class citizens. In particular, the notion of Business Artifact, initially proposed by Nigam and Caswell [15], opened the way to the development of a data-driven approach to the modeling of business operations. The data-driven approach counterposes a data-centric vision to the activity-centric vision, traditionally used when processes are explicitly modeled in terms of workflows. Business artifacts are concrete, identifiable, self-describing chunks of information, the basic building blocks by which business models and operations are described. They are business-relevant objects that are created and evolve as they pass through business operations. A business artifact includes an information model of the data, and a lifecycle model, the latter capturing the key states through which data, structured according to the information model, evolve together with state transitions. The lifecycle model is used both at runtime to track the evolution of business artifacts, and at design time to distribute tasks among the actors which operate on a business artifact. The presence of an explicit lifecycle gives business artifacts a semantics that differentiates them from other programming abstractions, like objects, active objects, and artifacts in the sense given to the concept by the A\&A meta-model [17]. The lack of autonomy differentiates them from agents. 
The work presented in this paper attacks a limit that business artifacts show: they do not provide the programmer with any means for designing and modularizing the coordination of those processes which should operate on them. It is a fact that business artifacts encapsulate data which are created and manipulated by many processes. For instance an order is created by a client who interacts with a seller, and is manipulated by the operations that make the transaction between the two proceed. The client, the seller, more in general the processes that operate on a business artifact need to agree on who should do what and sometimes even when to carry out a task of interest. For instance, payment and delivery are up to different parties in the purchase transaction, and they must both occur otherwise the transaction will not reach a happy end. There is a causal relationship between the actions of the merchant and of the client, the latter paying because of the promise (social engagement) of the merchant to deliver the goods.

So, a business artifact, besides having a lifecycle that describes its evolution from creation to some state where it is considered as archivable, is a natural candidate to be a medium of coordination and interaction. However, this latter dimension is not investigated by state-of-art literature on business artifacts.

The proposal we present in this paper aims at overcoming the described lack of business artifacts with a multiagent systems (MAS) approach (along the lines of activity theory, at the basis of A\&A, and exploiting commitments to represent social engagements). Specifically, we claim that: (1) services by which it is possible to operate on business artifacts should be encapsulated and organized into goal-oriented containers; (2) it is necessary to introduce a normative layer to capture the behaviors that are expected of the parties. The paper motivates our research and our proposal, and illustrates it with the help of a simple purchase example.

\section{Motivations}

In order to understand how business artifacts are currently specified and used, we briefly introduce the BALSA methodology [7] as a significant representative of the current approaches to business artifacts. The BALSA methodology specifies a data-centric declarative model of business operations, and can be summarized in three steps: 1) identify the relevant business artifacts of the problem at hand and their lifecycles, 2) develop a detailed specification of the services (or tasks) that will cause the evolution of the business artifact lifecycles, 3) define a number of ECA-rules (Event-Condition-Action) that create associations between services and business artifacts. ECA-rules are the building blocks to define, in a declarative way, processes operating on data.

BALSA (and similar) is extremely interesting, in particular because it introduces a novel perspective on the modeling of business processes. However, for what concerns coordination in presence of more business processes, the methodology simply refers to choreography languages and techniques proposed for service-oriented computing, despite the presence of the business artifacts which could themselves be natural instruments of coordination. The approach is sketched in Figure 1, where two business processes, $b p_{1}$ and $b p_{2}$, both defined declaratively in terms of ECA rules, access to the same business artifact. A proper synchronization between the services they invoke is 


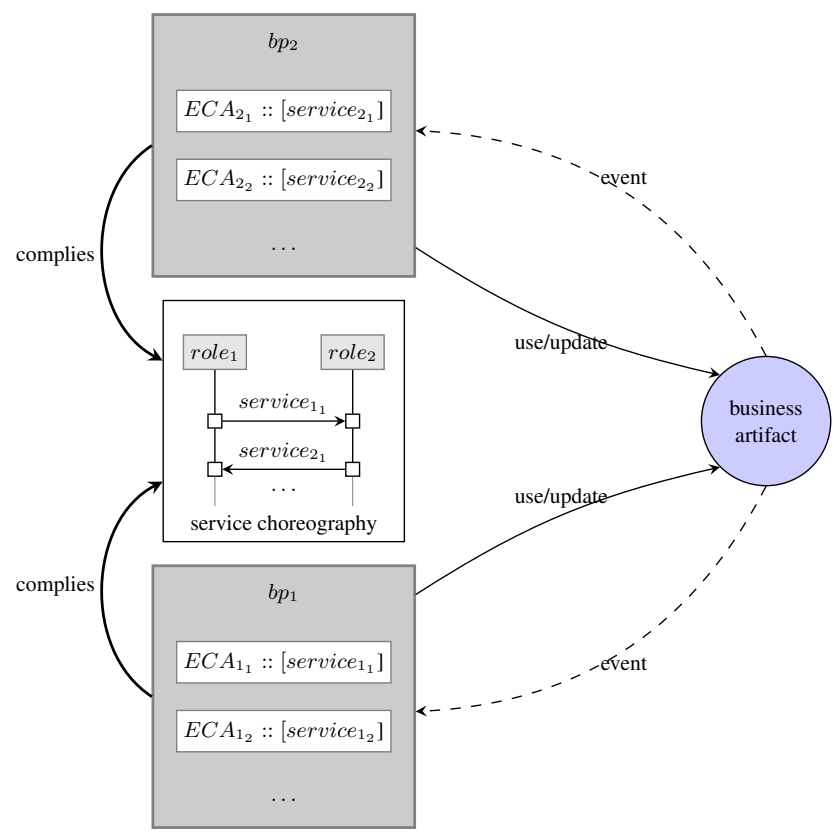

Fig. 1. Synchronized access to a business artifact via a choreography.

guaranteed by the fact that the two processes comply with a choreography specification.

We deem the absence of an explicit use of the business artifacts to the aim of coordination, which BALSA compensates by referring to the literature and the tools concerning choreographies, as a significant flaw. A choreography induces a form of subjective coordination [16]: each business process needs to include within its logic also the interaction logic that refers to the role it plays within the choreography. This lack of separation between the business and the coordination logic has two drawbacks: (1) it makes design and implementation more complex and reduces the possibility of reusing the same process in different contexts since it depends strictly on a specific choreography, and (2) it demands for an extensive compliance checking for guaranteeing that the process adheres to the choreography. In addition, in inherently destructured settings (e.g. cross-organizational settings), the involved actors are all peers, each of which has its own business goals, and acts in an autonomous way. Each actor does not know and does not care about the possible goals of others. They need, however, to interact to achieve goals they would not be able to achieve alone. This is the grounding of social engagements and causal relationships.

Interaction is, thus, a critical dimension that needs to be explicitly modeled to coordinate the usage of shared resources. This poses the question of how to scale the business artifact model to coordinate autonomous entities. We see in the introduction of a coordination model within business artifacts the way to achieve this goal, and explain what we mean with a simple example. 
Let us consider a purchase scenario, involving a merchant and a client. We claim that in order to coordinate the interaction between the two agents, it is necessary to add to the plain message exchange (which standard approaches to business processes envisage as the only means of interaction), one further abstraction that explicitly represents the engagements each player has towards the other. We also claim that business artifacts should trace such engagements and their evolution, in order to enable an effective agent coordination. For example, when offering to sell some goods, the merchant commits to the client to ship the items the client will pay for. Such a commitment is stored by the business artifact involved in the interaction between the two. Because of his awareness of such a commitment, the client, having paid for the goods, expects the shipment to occur. If this does not happen, the commitment progresses into a state of "violation" and this information, stored in the business artifact, provides a proof of the merchant's misbehavior. From a different perspective, a client is enticed to use a business artifact by the merchant's commitment, which makes explicit the course of interaction the merchant binds to, and creates a right on the client that such an expected course of action be respected (i.e., my payment will put an obligation on the merchant to ship the bought items or the merchant will violate the commitment). On the other hand, the merchant uses commitments inside the business artifact to entice interactions with potential clients - indeed, the obligation yielded by a commitment is activated only if a client pays for some goods.

In the example, the commitments that go along with a business artifact make explicit the behavior the agents are expected to stick to. They also have a normative flavour, as diverging behaviors will be considered as violations. This awareness causes agents to take part to an interaction only if they are fine with the commitments. As such, commitments provide a standard to define standards of interaction mediated by business artifacts. To realize this vision, we claim that: (1) services should be encapsulated and organized into goal-oriented containers; (2) it is necessary to introduce a normative layer. For what concerns (1), the Agent-Oriented Paradigm is a good candidate. In particular, the Agent and Artifact meta-model (A\&A) [17] has already shown how artifacts can be used as environment components that mediate agents' interactions. However, artifacts in the A\&A model are radically different from the business artifacts because they do not come with an explicit information model for data, and they do not exhibit data lifecycles. Thus, this information cannot be exploited at design time, nor at runtime, to reason about which actions an agent should take. Concerning (2), the normative layer would provide an explicit representation of the business artifacts lifecycles, and of how coordination is expected to occur. Such a representation would allow agents to reason about the use of business artifacts and to create mutual engagements for driving their activities. Indeed, we envisage engagements as encoding causal relations between the actions of an agent and the goals and actions of another, with a normative power that would allow each agent to have expectations on the behavior of the others. In the purchase example, it is easy to see how the introduction of a norm in form of the commitment whenever a customer pays, the merchant will ship the goods, would enhance coordination. The customer now knows that after service pay, the merchant will be pushed to consider the service ship-goods as one of its next goals because of the social engagement of the merchant with the customer. Should this not happen, the merchant 
would cause a violation. This provides the customer a guarantee about the achievement of its own goal (or to recoup its losses). An explicit normative layer plays a central role both at the design time, to verify whether all the engagements can converge towards their satisfaction, and at running time to monitor the execution of a system and determine the violation of engagements. In this paper we introduce the notion of normative business artifacts as a means to extend the artifact-centric approach with a normative layer, where engagements and norms are expressed in terms of social commitments [19]. The introduction of a normative layer in the more general setting of business processes is seen as desirable also in [21].

\section{Coordination via Normative Business Artifacts}

Business artifacts are, by definition, data-aware: they consider data as a first-class primitive that drives the construction of process models [6]. Business artifacts, however, are not an end in themselves: they are business relevant entities that are created, accessed, and manipulated by different services along a business process. We now show how to introduce a normative layer so that business artifacts support coordination.

Destructured business processes call for a modularization of the control flow. Agentoriented programming $[8,25]$ is conceived exactly for handling multiple and concurrent control flows. Two elements are central in agent-oriented programming: the agents and the environment. Agents, as abstractions of processes, possess their own control flow, summarized as the cyclic process in which an agent observes the environment (updating its beliefs), deliberates which intentions to achieve, plans how to achieve them, and finally executes the plan [8]. Beliefs concern the environment. Intentions lead to action [25], meaning that if an agent has an intention, then the expectation is that it will make a reasonable attempt to achieve it. In this sense, intentions play a central role in the selection and the execution of actions, which represent the innate capabilities agents have to modify their environment. Among others, (business) artifacts (see A\&A-meta model [17]) are privileged elements of an environment. In particular, in contexts where agents cannot achieve their goals on their own, but need to interact with other agents to do so, artifacts provide shared resources that agents will use to mediate their interactions.

We claim that business artifacts should be norm-aware in two ways. First, the lifecycle of a business artifact should be made explicit by way of norms that specify how data evolve. The agents (i.e., the artifact users), will be able to inspect and reason upon them to decide if and how to operate on an artifact to obtain some result. Second, agents need to coordinate and regulate their interaction while using the business artifacts to achieve their goals. Given these two bodies of norms, agents will apply reasoning techniques to plan proper coordination that, exploiting the social relationships and possibly without violating any norm, will lead to goal achievement. This is possible because norms enable the creation of expectations and commitments among agents.

Even though data-awareness and norm-awareness are by and large orthogonal to BDI [25] notions, it is natural to think of agents as BDI agents for a seamless integration of all the aspects of deliberation, including the awareness of data and of their lifecycles. For instance, an agent, that is involved in handling orders, may conclude that, since it has to pick up three items in the warehouse, since each such item is to be packed, since 




Fig. 2. Environment/Information system based on normative business artifacts.

all packagings are performed by a same other agent, and since one of its goals is saving energy, it is preferable to pick them up altogether, and deliver them to the other agent only afterwards, instead of picking and delivering one item at a time. Data-awareness here is awareness that three items of a same kind are requested. Norm-awareness that items are picked because each of them is part of some order, whose lifecycle says that after being picked they will be packed. Again data-awareness allows our agent to know that all parcels are to be made by a same other agent.

Relying on agent-oriented programming is promising also because the agent-based model allows to naturally tackle the issue of coordination by introducing the concept of norm [23]. The deliberative cycle of agents is affected by the norms and by the obligations these norms generate as a consequence of the agents' actions. The limit of current agent-based approaches is that they provide no holistic proposal where constitutive norms are used also to specify data operations, and where regulative norms are used to create expectations on the overall evolution of the system (agents behavior and environment evolution).

\subsection{Environment/Information systems based on normative business artifacts}

Figure 2 describes the high-level architecture of the kind of system we propose: (1) involving (normative) business artifacts and agents (with their goals), and (2) holistically norm-aware. Agents interact with each other and with the environment by creating and modifying data which belong to an information system and that are reified by business artifacts. They are goal-driven and capable of coordinating with other agents by creating and exploiting commitments, obligations, permissions, and prohibitions. The conceptual model of the information system is described in terms of the norms that regulate the evolution of data, that is, data lifecycles, capturing how data pass from one state to another as a consequence of actions that are performed by some agent. Moreover, business artifacts will include all those normative elements that regulate the coordination of the agents that interact by way of the artifact. This information is available to the interacting agents in a form that allows agents to reason on it. The agents are aware of the current state (of the lifecycle) of the data, as well as of the obligations, prohibitions, commitments, permissions put on them, and thus they are aware of the tasks expected 
of them and of their parties. At any time it is possible to check the execution, identifying pending tasks and who is responsible of them, as well as possible violations (e.g. of obligations or commitments), which may activate procedures specifically designed to handle the case.

Comparing our proposal with BALSA (outlined in Figure 1), we can highlight many advantages of using normative business artifacts, the first and most relevant one residing in the role played by a business artifact: in the BALSA meta-model, a business artifact is just a piece of data, or as pointed out in [15], the basis for factorization of knowledge that enables business operations. In our proposal, normative business artifacts become the media through which the interaction among processes (i.e., agents) happens. This idea has a software engineering solid foundation. In his survey on Concurrent ObjectOriented Languages (COOLs), Philippsen [18] highlights the importance of a locality principle for class correctness, and advocates that a way to achieve it is to realize a form of coordination on the side of the callee. Namely, the coordination is implemented in the class that is accessed concurrently. Moreover, Philippsen advocates that desirable properties of coordination code are isolation and separability. Isolation means that the code for coordination is isolated from the code that implements class functionality. Separability means that portion of the code for the coordination can be refined while other portions are reused. These two properties promote the modularity and reuse of code.

Normative business artifacts fall entirely in such a coordination model. The coordination is in fact implemented solely inside the artifact itself (i.e. the class called by the interacting parties). Consequently, coordination correctness can be assessed locally since all coordination code is part of the artifact implementation. It is no longer required to verify the compliance of agents towards a choreography, but it is sufficient to verify whether agents are capable of using an artifact. This can be done by verifying whether an agent has the capability of playing a role within an interaction, that is, if the agent can invoke (a subset of) role-dependent artifact operations to bring an interaction to conclusion [1].

In contrast to the service choreographies of the BALSA model, normative business artifacts induce a form of objective coordination [16], where coordination is addressed outside the interacting agents. Coordination is, thus, explicitly modeled as a first-class element of a MAS. It is responsibility of the coordination designer to identify the objectives of the coordination and the mechanisms through which the space of interactions can be manipulated by the agents. In other words, objective coordination enables a clear separation between the implementation of the business and the coordination logic by explicitly representing the environment where the agents operate. In our proposal, we meet this property by encapsulating a normative layer inside a business artifact. The resulting normative business artifact represents (a portion of) the environment within which the agents can interact by explicitly manipulating their engagements. Thus, the implementation of environment resources (i.e., artifacts) and of the agents can be carried out and verified in isolation.

Objective coordination also finds a justification within Activity Theory [14], which postulates that agents control their own behavior from the outside by using and creating "artifacts" through which they interact. In other words, according to activity theory the 
interaction between two agents is always mediated by a further element (e.g., a tool), that manipulated by an agent influences the other. This idea has inspired the A\&A metamodel [17] which, alongside agents, introduces the artifact abstraction as a building block of the agents' environment. According to A\&A, an artifact is a computational, programmable system resource that can be manipulated by the agents. Thus, an artifact becomes the means through which interaction actually occurs. Notably, A\&A relies also on programmable coordination media, that can for instance be the tuple centers adopted in TuCSoN [24], and derived by the notion of tuple space implemented in LINDA. The existence of a shared dataspace allows agents to coordinate directly on data. Communication becomes generative [9], in the sense that agents communicate by generating data in the dataspace, and these data are available to any agent having access to the dataspace. This vision is in contrast to the message passing paradigm, at the basis of choreographies, where communication is only enabled between agents sharing the same channel (typically, one sender and one receiver). In approaches based on tuple centers, data themselves become a coordination media.

We see the same potentiality in the business artifacts by Nigam et al. [15], but such a potentiality cannot be exploited within the BALSA methodology for two main reasons: 1) in the BALSA methodology coordination relies on service choreographies, that are based on message passing; and 2) the operations, that make the business artifact lifecycle evolve, are not associated with a precise operational semantics, upon which the coordination can be defined. The normative business artifacts we propose overcome these limitations. In fact, the introduction of a normative layer, based on commitments, allows us to associate operations, that cause the business artifact's progression along its lifecycle, with a semantics given in terms of commitment operations and state changes. Moreover, since commitments have a normative power, they allow the agents to create expectations about the behavior of the other parties, and hence they can be the building blocks upon which coordination can be explicitly defined. Finally, commitments are useful also at design time since they provide a programming interface between agents and their environment, given in terms of those state changes in the environment that are relevant to the agent and that the agent should tackle. This opens the way to agent programming methodologies as the CoSE methodology [2].

\section{Building Normative Business Artifacts in JaCaMo+}

In this section we explain how the normative business artifact we propose can be implemented by relying on the $2 \mathrm{COMM} / \mathrm{JaCaMo}+$ framework [3]. We refer to an implementation where the BDI agents are implemented in the Jason agent programming language, and where agents share artifacts, whose creation and manipulation involves an explicit creation and manipulation of social commitments [19]. Social commitments provide the normative layer and enable the coordination of the goal-driven agents.

We exemplify the implementation in the purchase scenario. In this scenario each agent has its own goals: the merchant has the goal of selling goods, while the customer has the goal of getting some goods. We show how they can achieve their goals by using a business artifact as the only means of coordination. To this end we need to present 


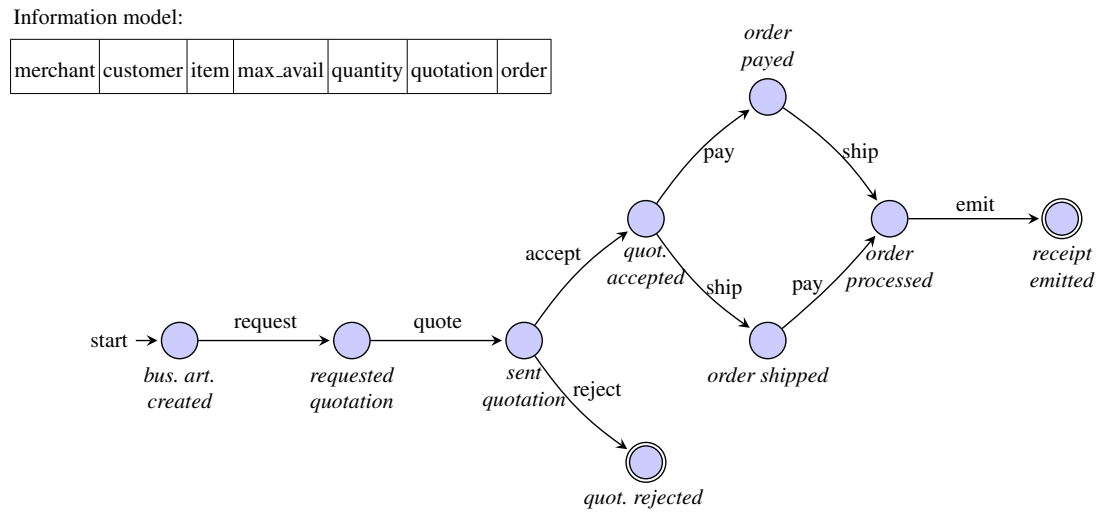

Fig. 3. The business artifact for the purchase scenario.

both sides of the interaction: the normative business artifact, on the one side, and how the agents use it, on the other side.

Let us consider the business artifact first. Figure 3 shows the business artifact which represents the transaction, occurring between a merchant and a customer. The specification of this business artifact follows the principles proposed in the BALSA meta-model: an information model specifies the relevant pieces of information (the merchant's and the customer's identifiers, the item sold by the merchant, and the maximum number of pieces that are available). While this information is provided at the time the business artifact is created, three further pieces of information (namely, quotation, quantity and order) are, instead, the result of the operations performed by the agents using the business artifact. These operations appear in the business artifact lifecyle showed here as an automaton: the customer asks the merchant for a quotation of a given quantity of items it wants to buy. Once the quotation is provided, the customer either decides to reject or accept the quotation. In the first case, the business artifact achieves a final state and can be archived. In the second case, a new order number is created to trace the shipping and the payment of the goods. Note that no order upon shipping and payment is imposed. After the payment, the merchant issues the payment receipt, and the business artifact can be archived.

As explained, such a business artifact is not sufficiently rich to trace the causal relationships captured by social engagements. The customer may have an expectation about the behavior of the merchant, raised by its experience in purchasing things, that the payment of an item will be followed by the merchant giving the item but this expectation has no normative power. We, therefore, extend this artifact with a normative layer that, as anticipated, is expressed in terms of a set of commitments. A social commitment $C(x, y, s, u)$ captures that agent $x$ (debtor) commits to agent $y$ (creditor) to bring about the consequent condition $u$ when the antecedent condition $s$ holds ( $s$ and $u$ are conjunctions or disjunctions of events). Only the debtor of a commitment can create it. When $s$ is true the commitment is detached and turns into an obligation on the debtor. When $u$ 
is true the commitment is satisfied. A detached commitment that is canceled or whose consequent becomes false is violated.

To realize a normative business artifact, thus, it is sufficient to associate each operation on the business artifact with operations (e.g. create, discharge, etc.) on one (or more) commitment(s). It follows that a normative business artifact, besides representing the chunk of information at hand, maintains also the created commitments, that can be inspected by the agents. Specifically agents will be notified of the changes to the business artifact state which include changes occurred to the commitments. Among other events, they will be aware of the detachment of commitments of which they are debtors, and of the satisfaction (violation) of commitments of which they are creditor.



Listing 1.1. The normative layer in the purchase scenario

In our example, the normative layer is given by the set of commitments in Listing 1.1. For the sake of readability, the commitments are expressed following the syntax of the Cupid language [11]. The first commitment, ShipGoods, is created by the merchant when it executes the quote operation. That is, besides giving a value to the quotation information, the quote operation also commits the merchant towards the customer. Such a commitment is discharged when the customer accept the quotation, and discharged when the merchant ships the bought goods to the customer. Also the second commitment, EmitReceipt, is created by the merchant by the execution of the quote operation. In this case, the commitment is detached when the customer pays for the goods, and it is discharged when the merchant emits the receipt towards the customer. Both these two commitments are released by the customer when it rejects the quotation provided by the merchant. The last commitment, PayForGoods, is created by the customer when it accepts the merchant's quotation. The commitment is detached when the merchant has shipped the goods, and discharged when the customer pays for them.

Now, let us briefly review the resulting normative business artifact. As before, the merchant advertises, by creating the artifact, some item to be sold, and specifies the number of available units. An interested customer, by inspecting the artifact, can now see the commitments that the merchant is willing to take towards the customer. That is, the customer can create expectations about the merchant's behavior that have a normative power. The customer is, thus, enticed to accept the quotation because of the presence of the commitment, as part of the information provided by the business artifact. This, indeed, yields that this action will create an obligation on the merchant to deliver the goods that will make it achieve his goal. On the other hand, the customer will 
see also the commitments it will take in favor of the merchant, should it join the interaction. So, if the customer starts an interaction by requesting a quotation for a number of units, the merchant will provide such a quotation and, at the same time, it will create a commitment to: 1) ship the goods, provided that the customer accepts the quotation (ShipGoods), and 2) emit a receipt upon the payment for the goods (EmitReceipt).

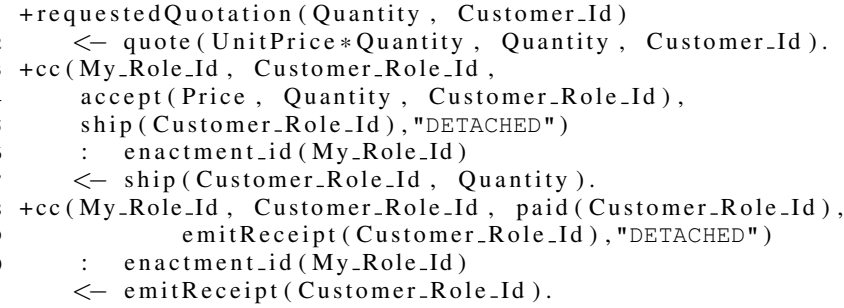

Listing 1.2. Excerpt of the merchant program.

Note how the operations performed by agents on the business artifact make the commitments progress. For instance, the customer's acceptance of the quotation has several effects: 1) on the data side, a new order number is created to trace shipping and payment; 2) the customer commits towards the merchant to pay for the goods once they are delivered (PayForGoods); 3) commitment ShipGoods is detached, and then the merchant is now asked to ship the goods. As a final comment about the artifact, note how the commitments do not impose any ordering about the payment and shipping. In fact, the customer could pay as soon as it accepts the quotation, assured by the existence of commitment ShipGoods that pushes the merchant to actually ship the goods.

A natural way to implement normative business artifacts is to rely on the $2 \mathrm{COMM}$ framework [3]. In 2COMM normative business artifacts are reified as commitmentbased protocol artifacts, that are provided by the framework as Java classes. Business artifact operations are mapped into protocol actions, whereas the data dimension is captured by the notional social state that protocol artifacts maintain. Indeed, the social state kept by a protocol artifact traces both the data required for the interaction, and the commitments, together with their states, that are created and manipulated along the interaction.

Let us now discuss the other side of the interaction, that is, how the agents use a normative business artifact. To exemplify the agents, we use here the JaCaMo+ framework, consisting in the well-known JaCaMo multi-agent platform enriched with commitment protocols provided by means of the 2COMM framework. Listing 1.2 reports an excerpt of the merchant agent program. In this first plan, the merchant is solicited to act by the reception of a requestedQuotation event, that comes from a customer through the business artifact. The body of the plan consists in the execution of quote, which sends a quotation to the customer and causes the creation of the merchant's commitments ShipGoods and EmitReceipt. The second plan captures the detachment of the ShipGoods commitment. The detach of the commitment is indeed an event generated by the ar- 
tifact the merchant is focusing on, and it is the consequence of the accept operation performed by the customer. The body of the plan consists in the ship operation. Finally, the third plan captures the detachment of the EmitReceipt commitment, also in this case the body of the plan aims at discharging the commitment.

This example shows how the two agents, merchant and customer, can interact with each other by using a business artifact as an interaction medium. The normative layer enables each agent to achieve its own goals by relying on the others. The commitments, in fact, provide an explicit representations of the social engagements existing between the two agents which, behaving so as to accomplish their duties, bring about their goals (and help others to achieve theirs). Without commitments, agents could only rely on the lifecycle of the business artifact, that discloses the possible evolution of the data, but only when the lifecycle is complemented by an explicit representation of the social engagements an agent knows that by performing an action it will create an expectation on the side of the counterpart (and vice versa). In other words, the lifecycle traces the data dimension, and this dimension is orthogonal to the goal/process dimension which is captured by the agents. The normative layer operates as a sort of glue between the dimensions. This is also apparent in the lifecycle in Figure 3. The two final states model conditions in which the business artifact can be archived independently of the status of the agents using it, that is, independently of whether they have achieved their goals. Looking at the normative layer, however, one can give them a proper semantics in terms of commitment states and goals. One further advantage of adding the normative layer is the possibility of making explicit some of the goals and services agents make available to others. This piece of knowledge can be used by the agents in a practical reasoning step (see [22]) to decide whether to join the artifact.

\section{Conclusions}

The presented work is strictly related to the problem of interaction in multiagent systems. In these systems, interaction is mainly focused on the modeling of communication patterns (protocols), which are concerned with the sequence of messages that can be exchanged between two communicating agents, but disregard the information conveyed by these messages. Recent approaches such as HAPN [26] and BSPL [20] have started to consider also the information dimension. HAPN is formally based on automata where nodes represent states of the interaction and transitions between nodes represent the messages that can be exchanged. Transitions have a complex structure since for each message it is possible to define a guard condition on message sending. A similar approach is BSPL where the information flow is decomposed in a number of "simple protocols", each defining the schema of the messages that can be exchanged together with their parameters. Parameter are decorated as in or out (meaning it is received or emitted). BSPL provides a formal framework in which it is possible to verify properties such as liveliness and safety of a protocol. Both HAPN and BSPL, however, show some weaknesses in properly handling information. In HAPN, for instance, guards, that enable message sending, may refer to information which is not carried by the message itself, but rather maintained in an external information system, which is not an integral part of the HAPN proposal, and hence the complete verification of an 
interaction is not actually achievable. BSPL, on the other hand, assumes a distributed view of information. Each participant has its own knowledge base, and the progression of the interaction makes the local knowledge bases evolve. The problem, in this case, is that each participant has just a local view of the information lifecycle. Thus, an agent cannot create expectations about the behaviors of other participants as a consequence of the messages it sends. The approach we propose overcomes these limitations. Business artifacts abstract an information system, and provide the environment in which the agents, which are autonomous loci of control, interact. Both business artifacts and agents are first-class components. The autonomy and flexibility of the agents are preserved and supported; moreover, it is possible to reason both on the evolution of the business artifacts and on the interaction. This work can be extended along three main lines of research. First of all, an explicit normative layer paves the way to formal verification techniques for cross-organizational business processes. In this respect, the notion of accountability is rapidly gaining importance since, when more organizations come into play, it is even more important to trace back who is responsible for what. First steps can be found in [4]. Another promising extension is to understand how agents could plan the use of business artifacts for reaching their goals. An initial attempt to use social commitments in planning has been discussed in [5], but business artifacts are yet to be considered. Finally, the standardized lifecycle of commitments can be the key for developing an agent programming methodology, similar to the one discussed in [2]. The idea is to program agents so that they can properly tackle part of the events that are generated in the business artifacts of their interest; specifically, the state transitions that occur to commitments in which they are involved. To conclude, we mention RAW-SYS [13], which enriches the prescriptive process model with data-awareness. Although RAW-SYS looks similar to a (normative) business artifact, the objectives of the two models are quite different. RAW-SYS is essentially a framework for verifying business processes taking into account both the control- and the data-flows. A normative business artifact, instead, aims at coordinating autonomous agents.

Acknowledgements This work was partially supported by the Accountable Trustworthy Organizations and Systems (AThOS) project, funded by Università degli Studi di Torino and Compagnia di San Paolo (CSP 2014). The authors warmly thank the reviewers for their constructive and helpful comments which helped revising the paper.

\section{References}

1. Matteo Baldoni, Cristina Baroglio, and Federico Capuzzimati. Typing Multi-Agent Systems via Commitments. In F. Dalpiaz, J. Dix, and M. B. van Riemsdijk, editors, Post-Proc. of the 2nd International Workshop on Engineering Multi-Agent Systems, EMAS 2014, Revised Selected and Invited Papers, number 8758 in LNAI, pages 388-405. Springer, 2014.

2. Matteo Baldoni, Cristina Baroglio, Federico Capuzzimati, and Roberto Micalizio. Empowering Agent Coordination with Social Engagement. In M. Gavanelli, E. Lamma, and F. Riguzzi, editors, $A I^{*} I A$ 2015: Advances in Artificial Intelligence, XIV International Conference of the Italian Association for Artificial Intelligence, volume 9336 of LNAI, pages 89-101, Ferrara, Italy, September 2015. Springer. 
3. Matteo Baldoni, Cristina Baroglio, Federico Capuzzimati, and Roberto Micalizio. Commitment-based Agent Interaction in JaCaMo+. Fundamenta Informaticae, 2017. To appear.

4. Matteo Baldoni, Cristina Baroglio, Katherine M. May, Roberto Micalizio, and Stefano Tedeschi. ADOPT JaCaMo: Accountability-Driven Organization Programming Technique for JaCaMo. In PRIMA 2017: Principles and Practice of Multi-Agent Systems, 20th International Conference, number 10621 in LNCS, pages 1-18, Nice, France, 2017. Springer.

5. Matteo Baldoni, Cristina Baroglio, and Roberto Micalizio. Social Continual Planning in Open Multiagent Systems: a First Study. In Q. Chen, P. Torroni, S. Villata, J. Y. Hsu, and A. Omicini, editors, PRIMA 2015: Principles and Practice of Multi-Agent Systems, 18th International Conference, number 9387 in Lecture Notes in Computer Science, pages 575584, Bertinoro, Italy, October 26th-30th 2015. Springer.

6. Kamal Bhattacharya, Nathan S. Caswell, Santhosh Kumaran, Anil Nigam, and Frederick Y. $\mathrm{Wu}$. Artifact-centered operational modeling: Lessons from customer engagements. IBM Systems Journal, 46(4):703-721, 2007.

7. Kamal Bhattacharya, Richard Hull, and Jianwen Su. A data-centric design methodology for business processes, pages 503-531. Handbook of Research on Business Process Modeling. IGI Publishing, 2009.

8. Michael E. Bratman. What is intention? In P. Cohen, J. Morgan, and M. Pollack, editors, Intensions in Communication, pages 15-31. MIT Press, Cambridge, MA, 1990.

9. Nadia Busi, Paolo Ciancarini, Roberto Gorrieri, and Gianluigi Zavattaro. Coordination Models: A Guided Tour, pages 6-24. Springer Berlin Heidelberg, 2001.

10. Diego Calvanese, Giuseppe De Giacomo, and Marco Montali. Foundations of data-aware process analysis: a database theory perspective. In Proceedings of the 32nd ACM SIGMODSIGACT-SIGART Symposium on Principles of Database Systems, PODS, pages 1-12. ACM, 2013.

11. Amit K. Chopra and Munindar P. Singh. Cupid: Commitments in relational algebra. In Blai Bonet and Sven Koenig, editors, Proceedings of the Twenty-Ninth AAAI Conference on Artificial Intelligence, January 25-30, 2015, Austin, Texas, USA., pages 2052-2059. AAAI Press, 2015.

12. David Cohn and Hull Richard. Business Artifacts: A Data-centric Approach to Modeling Business Operations and Processes. IEEE Data Eng. Bull., 32(3):3-9, 2009.

13. Riccardo De Masellis, Chiara Di Francescomarino, Chiara Ghidini, Marco Montali, and Sergio Tessaris. Add data into business process verification: Bridging the gap between theory and practice. In Proceedings of the Thirty-First AAAI Conference on Artificial Intelligence, February 4-9, 2017, San Francisco, California, USA., pages 1091-1099, 2017.

14. Yrjö Engeström, Reijo Miettinen, and Raija-Leena Punamäki, editors. Perspectives on Activity Theory. Cambridge University Press, Cambridge, UK, 1999.

15. Anil Nigam and Nathan S. Caswell. Business artifacts: An approach to operational specification. IBM Systems Journal, 42(3):428 - 445, 2003.

16. Andrea Omicini and Sascha Ossowski. Objective versus subjective coordination in the engineering of agent systems. In AgentLink, volume 2586 of Lecture Notes in Computer Science, pages 179-202. Springer, 2003.

17. Andrea Omicini, Alessandro Ricci, and Mirko Viroli. Artifacts in the A\&A meta-model for multi-agent systems. Autonomous Agents and Multi-Agent Systems, 17(3):432-456, December 2008. Special Issue on Foundations, Advanced Topics and Industrial Perspectives of Multi-Agent Systems.

18. Michael Philippsen. A survey of concurrent object-oriented languages. Concurrency - Practice and Experience, 12(10):917-980, 2000.

19. Munindar P. Singh. An ontology for commitments in multiagent systems. Artif. Intell. Law, 7(1):97-113, 1999. 
20. Munindar P. Singh. Information-driven interaction-oriented programming: BSPL, the blindingly simple protocol language. In 10th International Conference on Autonomous Agents and Multiagent Systems (AAMAS), pages 491-498, 2011.

21. Munindar P. Singh. NoBPM: Supporting Interaction-Oriented Automation via Normative Specifications of Processes, 2015. Invited talk, BPM.

22. Pankaj R. Telang, Munindar P. Singh, and Neil Yorke-Smith. Relating Goal and Commitment Semantics. In Post-proc. of ProMAS, volume 7217 of LNCS. Springer, 2011.

23. Göran Therborn. Back to norms! on the scope and dynamics of norms and normative action. Current Sociology, 50:863-880, 2002.

24. Mirko Viroli, Andrea Omicini, and Alessandro Ricci. Infrastructure for RBAC-MAS: an approach based on agent coordination contexts. Applied Artificial Intelligence, 21(4\&5):443467, 2007.

25. Michael J. Wooldridge. Introduction to multiagent systems, 2nd edition. Wiley, 2009.

26. Nitin Yadav, Lin Padgham, and Michael Winikoff. A tool for defining agent protocols in HAPN: (demonstration). In Proceedings of the 2015 International Conference on Autonomous Agents and Multiagent Systems, AAMAS, pages 1935-1936, 2015.

\section{A An Example of Enhanced Business Artifact}

\section{A.1 merchant.asl}

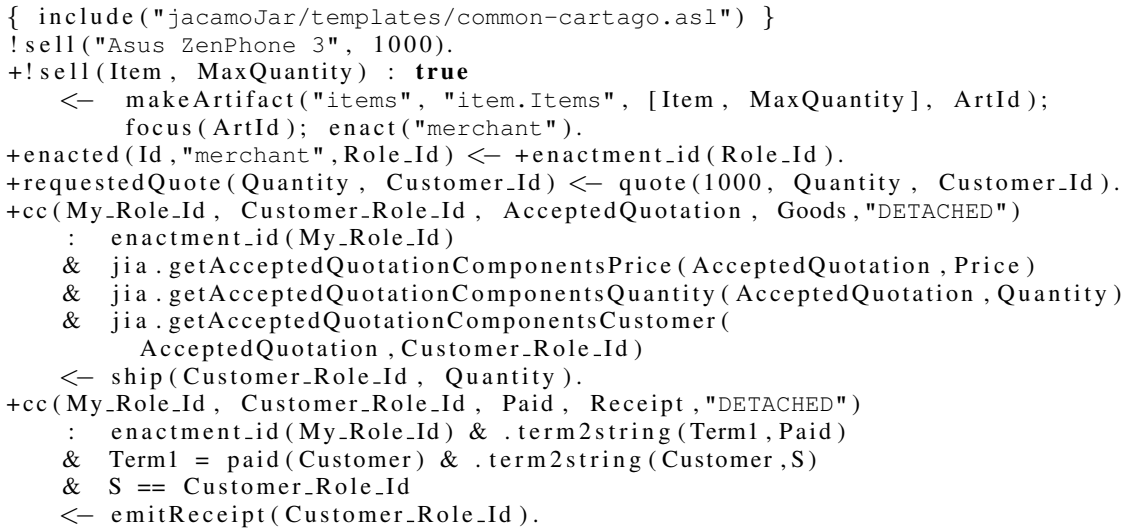

\section{A.2 customer.as}






\section{A.3 Items.java}

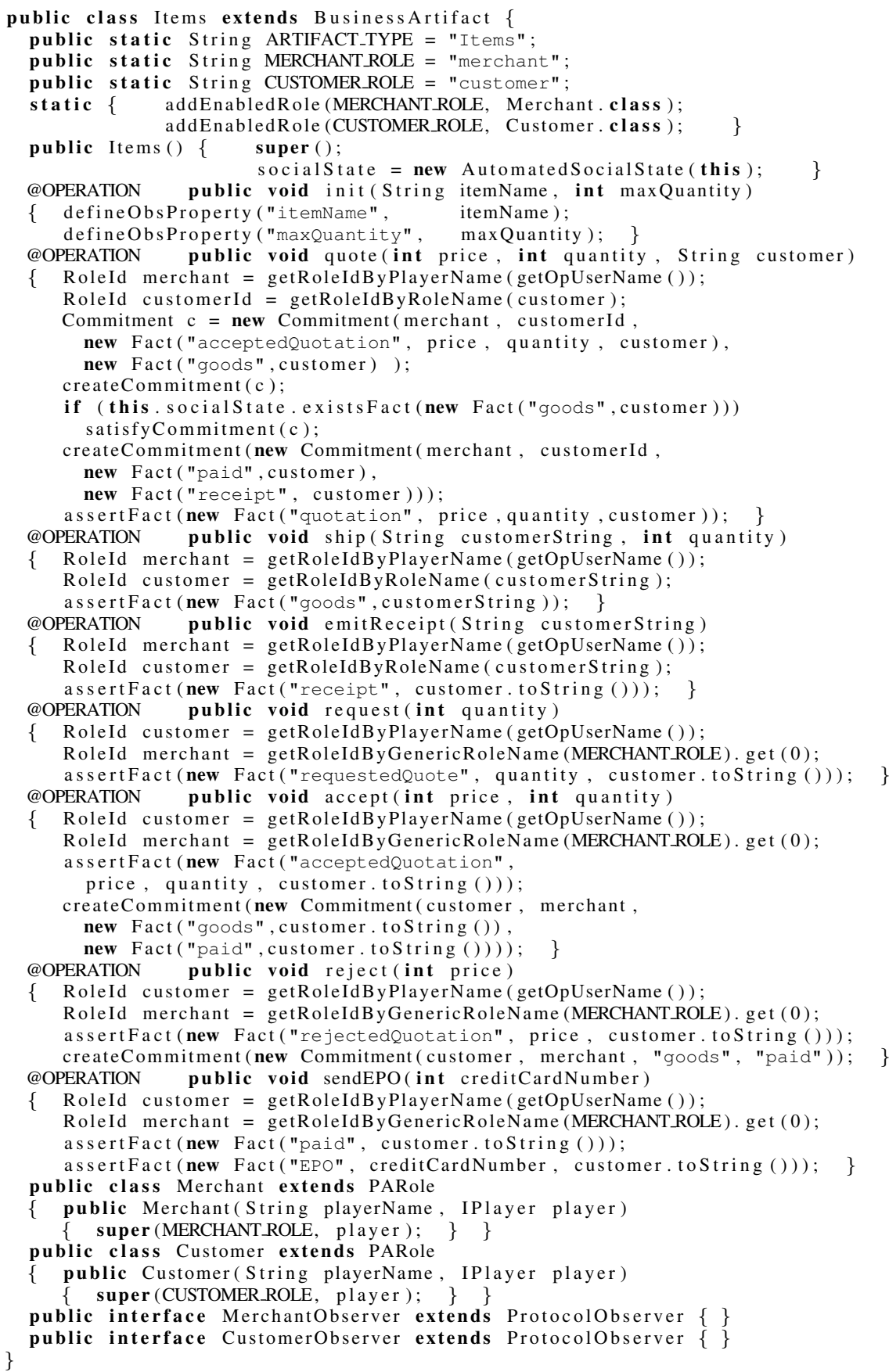

